\title{
The Water Molecule with Conjoint Translational, Rotational and Spin Degrees of Freedom in the Gas and Liquid Phases
}

\author{
V.K.Konyukhov \\ A. M. Prokhorov General Physics Institute, Russian Academy of Sciences, Vavilov Street 38, 119991 Moscow, Russia \\ Corresponding Author: collie@kapella.gpi.ru
}

Copyright (C)2014 Horizon Research Publishing All rights reserved.

\begin{abstract}
The theoretical model of water molecule is created which gives an opportunity to understand what happen with translational, rotational and spin degrees of freedom when molecule makes transitions from gas into liquid phase and vice versa. Translational degrees of freedom are considered classically but rotational and spin degrees of freedom are treated according to quantum-mechanical rules. The model is based on the mathematical techniques of geometric algebras over the field of real numbers.

Unusual properties of water molecules with conjoint degrees of freedom compared to usual or free molecules are predicted and denoted as latent in future. The latent molecules have not the full rotational spectrum but possess three isolated rotational levels only. The latent molecules are capable of moving along straight lines at finite distances in 3D-space. The latent molecules conserve all their degrees of freedom during the transition from gas into liquid phase.
\end{abstract}

Keywords ortho-water, para-water, adsorption, gas-phase reaction, gas-liquid transition, geometric algebras

\section{Introduction}

Water molecules continue to attract an permanent attention of scientists due to an exceptionally significant role that this molecule plays in the existence of living things and technological processes. The behavior of low-energy degrees of freedom, namely rotational, translational and spin ones constitute a complex and unsolved today problem if we deal with the transfer of molecule from gas into liquid phase or adsorbed state. Complications arise since these degrees of freedom may be linked with each other by a correlative prohibition and symmetrical selection rules that continue to be right during gas-liquid transition. The most striking example of such behavior of water molecules is the spin-selective adsorption on solid surfaces with appearing the spin-isomer non-equilibrium states in water vapor.

It is proposed usually that molecules lose their rotational, translational and spin degrees of freedom under phase transition. More correctly, the given degrees of freedom integrate with the corresponding degrees of freedom of condensed matter while electronic and vibrational degrees of freedom continue to belong to a single molecule. If we take this point of view and suppose that it is right for the rotational degrees of freedom of water molecule then we have a problem: how an ortho-molecule transfers into adsorbed state with losing rotational angle momentum? Note, that the quantum number of angle momentum of ortho-molecule in ground state is equal to 1 (according to classical mechanics we have rotating molecule!), while the momentum number of para-molecule in the ground state is equal to 0 and we have not any problem with adsorption of this isomer. The ortho- molecule can not make a transition to the ground state of the para-molecule due to the strong quantum prohibition based on the Pauli exclusion principle.

There is another problem related to the likeness and distinction of water and hydrogen molecules. In regard to their spin degrees of freedom they are looking the same, because they have two protons with half-spin moments. However, the rotational functions of hydrogen molecule are defined on the $S^{2}$ in 3D-space (there are two angular variables $\theta, \phi$ ) while the rotational functions of water molecule are defined on the $S^{3}$ in 4D-space (there are three angular variables $\alpha, \beta, \gamma$ ). We deal here with the Wigner functions [35]. Therefore the problem of the quantum asymmetric rotor must be solved by means of methods which are suitable to the Euclidean 4Dspace.

The conventional approach to the consideration of problems of quantum rotors avoids this complication by using the so(3) algebra of angle momentum operators in both cases of hydrogen and water molecules. It should be recalled that in case of asymmetric rotors the series of unitary matrix representations of $\mathrm{SU}(2)$ group are used with the integer $\mathrm{L}=0,1,2,3$. angle momentum. Matrix elements of any rows or columns of the representation matrix $(2 L+1) \times(2 L+1)$ can be used as basis vectors on the Hilbert space, therefore its dimension is $2 \mathrm{~L}+1$ in general case. Three matrices that represent the so(3) algebra are defined on the Hilbert space taking into account the functional form of basis vectors. After that the rotational Hamiltonian is created. The diagonalization of the Hamiltonian provides rotational levels and their wave functions. This approach for describing the rotation of polyatomic molecules has a rich history [15] [10] [27] [29] [12]. 
Such type of rotational states will be denoted in what follows as the free rotation.

The task of asymmetric quantum rotors has another problem that is regarded evident and have not been considered. There are two Cartesian coordinate systems in 3D-space one of which is associated with the nuclear frame of molecule. The principal moments of inertia are calculated using this reference system, values of which will be inserted as the scalar dimensional coefficients into the rotational Hamiltonian of molecule. The other system is connected with macroscopic objects that are parts of the molecular environment. The basis vectors of two coordinate systems can algebraically be written into each other using the $\mathrm{SO}(3)$ matrix of all rotations on 3D-space. It is making so in a case, when the probability of dipole transition between rotational levels must be calculated assuming that an external electrical field is defined in the laboratory system and a dipole moment in molecular one. The operators of dipole moment are linear combinations of the matrix elements of $\mathrm{SO}(3)$.

The reasoning given above has a certain contradiction that remains without proper attention. The first reference system belongs to a microscopic object, therefore its basis vectors must have the length that is in agreement with dimensions of an object. The second one has the basis vectors of macroscopic dimensions, but both coordinate systems are used as fully equivalent objects.

The transition to the so(4) algebra or quaternion algebra has a negative side because the complex numbers are excluded for all operations. The group $\mathrm{SO}(4)$ and its algebra are defined for the field of real numbers. As soon as a wave function will be appeared from calculations it will be the real function of its variables and therefore it will not contain the phase factor that is the important quantum-mechanical object. The lack of the phase factor one can overcome by means of introduction a conjugate quaternion that is the analog of complex conjugate wave function. Recall that the multiplication of given quaternion and its conjugate one is a real number that is analog of the square module of wave function [2]. The transfer from complex numbers to real ones must be made in the representation matrices of $\mathrm{SU}(2)$ group by extracting a real part of matrix elements. However, this operation will be made with another method that will be described below. It should be noted that the change of complex numbers into real ones does not affect the logic of quantum mechanics, therefore such transformation is allowed [4].

The conjugate quaternion has a physical interpretation as a reverse rotation. If we have two coordinate systems as mention above then the rotation of the most general form has three rotations $O z(\alpha), O y(\beta), O z(\gamma)$ around the axes of the laboratory coordinate system. Rotational angles are given in brackets [31]. To return the molecular system to its initial state it is necessary not only to have the angles with an opposite sign, but to change also the sequence of the execution of rotations. The reverse rotation is written as $O z(-\gamma), O y(-\beta), O z(-\alpha)$. It's clear, that the reverse rotation differs from direct one by the mathematical and physical sense [16]. The introduction of reverse rotation in the case of free water molecules does not give any new rotational states because the mathematical description of rotation does not change.

New mathematical methods are introduced which are known as the geometric or Clifford algebras, in particular the algebra $\mathcal{G}\left(R^{3,0}\right)$ over the field of real numbers [9], [25],
[13]. This innovation is based on an advantage that the single mathematical construction spans the translational, rotational and spin degrees of freedom of molecule. Each of three types of movements undergoes qualitative modifications. The molecular center of mass has the capability to move, according to rules of classical mechanics, along only straight lines in 3D-space. Quantum rotational energy states of molecule lose their $2 \mathrm{~L}+1$ degeneracy corresponding to the projection on the $\mathrm{z}$ axis of laboratory coordinate system. The concept of the nuclear spin symmetry peculiar to the spin system of free molecule disappears since the superposition of states of two spin moments known as singlet and triplet with $\mathrm{m}=0$ do not exist now.

The basis elements $\mathbf{e}, \mathbf{e}^{\mathbf{1}}, \mathbf{e}^{\mathbf{2}}, \mathbf{e}^{\mathbf{3}}$ of algebra $\mathcal{G}\left(R^{3,0}\right)$ are represented by Pauli matrices, elements $\mathrm{e}^{23}, \mathrm{e}^{31}, \mathrm{e}^{12}$ by unitary matrices of quaternions [1], a quasiscalar $\mathbf{e}^{\mathbf{1 2 3}}$ is represented by matrix diag(i,i). To confirm the validity of all operations which are defined for algebra $\mathcal{G}\left(R^{3,0}\right)$ the next operations have to execute with matrices of representation (i) for geometric or Clifford multiplication is used conventional matrix multiplication, (ii) for outer multiplication one needs to calculate a commutator, (iii) for inner multiplication one needs to calculate an anticommutator (see Appendix A).

The introduction of algebra $\mathcal{G}\left(R^{3,0}\right)$ radically changes the behavior of molecules and the problem obtains some attributes of the relativism by remaining at the same time in 3Dspace. If a multiplication table of basic elements of $\mathcal{G}\left(R^{3,0}\right)$ algebra was calculated, using outer multiplication only (the commutator of elements), we obtain a Lee algebra of $\mathcal{G}\left(R^{3,0}\right)$ which is isomorphic to the Lee algebra of Lorentz group. This is a reason that allows one to say that algebra has relativistic features. Details are on Appendix B.

The second relativistic feature of $\mathcal{G}\left(R^{3,0}\right)$ algebra consists in the required existence of the coordinate system or reference system. In our case elements $\mathbf{e}^{1}, \mathbf{e}^{2}, \mathbf{e}^{3}$ play a role of the macroscopic vectors of reference system in the laboratory environment. Elements $\mathrm{e}^{23}, \mathrm{e}^{31}, \mathrm{e}^{12}$ play role of the microscopic planes of reference system obliged to molecules. The dimensional difference between vectors and planes can be excluded by means of the dimensionless factor of $10^{-9}$ which belongs to $\mathrm{e}^{\mathbf{1 2 3}}$ element. This condition is equivalent to that one as if vectors to be measured in CGS units but plates in atomic units.

There are several ways to introduce coordinates into the reference system that is obliged to $\mathcal{G}\left(R^{3,0}\right)$ algebra. We take an advantage of one that is more intuitive and the most convenient. First, it should be noted that the quaternion algebra has some likeness to the vector field of geometric $\mathcal{G}\left(R^{3,1}\right)$ algebra. In both cases there are 4 basic vectors among which we can choose one vector which is distinguished by its properties. In case of quaternion algebra there is the unit vector 1 commuting with all elements of algebra. In case of $\mathcal{G}\left(R^{3,1}\right)$ algebra it is the basic vector having the square with the different sign compared to the sign of three other basic vectors. We realize that this is a formal resemblance which has not any implications to other properties of algebras. But this trick enable us to transfer coordinate variables $x_{0}, x_{1}, x_{2}, x_{3}$ belonging to any quaternion to basic vectors of $\mathcal{G}\left(R^{3,1}\right)$ algebra. After that bivectors of $\mathcal{G}\left(R^{3,1}\right)$ algebra obtain the proper coordinates in the form of paired multiplication according to the generation rule of bivectors. The indexes of coordinate and of proper bivector are fully coincide according to their definition. Finally, we can use the isomorphism that exist between 
the subalgebra $\mathcal{G}^{+}\left(R^{3,1}\right)$ and $\mathcal{G}\left(R^{3,0}\right)$ algebra to transform coordinates of bivectors to the elements of reference system (see Appendix A).

So we obtained the reference system composed of three vectors and three planes with scalar real variables performing role of coordinates and of wave functions as will be displayed later.

$$
x_{0} x_{1} \mathbf{e}^{\mathbf{1}}, x_{0} x_{2} \mathbf{e}^{\mathbf{2}}, x_{0} x_{3} \mathbf{e}^{\mathbf{3}}, x_{2} x_{3} \mathbf{e}^{\mathbf{2 3}}, x_{1} x_{3} \mathbf{e}^{\mathbf{3 1}}, x_{1} x_{2} \mathbf{e}^{\mathbf{1 2}}
$$

Constructions composed of basis vectors, basis planes and coordinates have been considered on [26].

\section{Rotational Hamiltonian, energy levels}

To construct a rotational Hamiltonian we will use an approach peculiar to the case of free rotation of molecule and will have to restrict to the so(3)algebra. The paired variables $x_{2} x_{3}, x_{1} x_{3}, x_{1} x_{2}$ can be treated as rotational functions because the elements $\mathrm{e}^{23}, \mathrm{e}^{31}, \mathrm{e}^{12}$ of reference system are commuted as the elements of so(3) algebra. The Hermit operators of angular momentum on a Hilbert space will be the next differential operators [32]

$$
\begin{aligned}
& L_{23}=i \hbar\left(x_{3} \frac{\partial}{\partial x_{2}}-x_{2} \frac{\partial}{\partial x_{3}}\right) \\
& L_{31}=i \hbar\left(x_{1} \frac{\partial}{\partial x_{3}}-x_{3} \frac{\partial}{\partial x_{1}}\right) \\
& L_{12}=i \hbar\left(x_{2} \frac{\partial}{\partial x_{1}}-x_{1} \frac{\partial}{\partial x_{2}}\right)
\end{aligned}
$$

The Hamiltonian mentioned above has the same rotational constants $c<b<a$ as in the case of free rotation of molecule.

$$
\begin{gathered}
\mathcal{H}=c \hat{L}_{23}\left(\hat{L}_{23}(\psi)\right)+b \hat{L}_{31}\left(\hat{L}_{31}(\psi)\right)+a \hat{L}_{12}\left(\hat{L}_{12}(\psi)\right) \\
\hat{L}_{j k}=L_{j k} / i \hbar \\
\psi=\operatorname{Span}\left\{x_{2} x_{3}, x_{1} x_{3}, x_{1} x_{2}\right\}
\end{gathered}
$$

The paired variables $x_{2} x_{3}, x_{1} x_{3}, x_{1} x_{2}$ turn out to be eigen functions of the Hamiltonian with eigen values $4 \mathrm{c}+\mathrm{b}+\mathrm{a}$, $\mathrm{c}+4 \mathrm{~b}+\mathrm{a}, \mathrm{c}+\mathrm{b}+4 \mathrm{a}$, where $c=\hbar^{2} / I_{c}$ is the inertia moment with respect to the axis that is vertical to the molecular plane. Also $b=\hbar^{2} / I_{b}$ is the inertia moment with respect to the symmetry axis of molecule and $a=\hbar^{2} / I_{a}$ is the inertia moment with respect to the third principal axis of inertia. The rotational constants $c=9.287, b=14.507, a=27.876$ provide the energies $79.531,95.191,135.298$ of rotational levels in $\mathrm{cm}^{-1}$ unit. Those energies coincide with the energy of levels $2_{21}, 2_{11}, 2_{12}$ of free molecule because we have assumed that the rotational constants are the same.

The basic vectors $\mathbf{e}^{\mathbf{1}}, \mathbf{e}^{\mathbf{2}}, \mathbf{e}^{\mathbf{3}}$ do not form an algebra therefore we can not treat coordinates $x_{0} x_{1}, x_{0} x_{2}, x_{0} x_{3}$ as wave functions. We will have to consider the elements $x_{0} x_{1} \mathbf{e}^{\mathbf{1}}, x_{0} x_{2} \mathbf{e}^{\mathbf{2}}, x_{0} x_{3} \mathbf{e}^{\mathbf{3}}$ as positions of molecule on straight lines in 3D-space directed by $\mathbf{e}^{\mathbf{1}}, \mathbf{e}^{\mathbf{2}}, \mathbf{e}^{\mathbf{3}}$ vectors.

It should be noted, that there are three different operations, namely (i) the reverse rotation replaces the direct rotation, (ii) the conjugation of quaternion, (iii) the inversion on 3D-space have the identical mathematical form that variables $x_{1}, x_{2}, x_{3}$ obtain an opposite sign. We can divide the basic elements of reference system into two sets according to the operations mentioned above. The elements $x_{0} x_{1} \mathbf{e}^{\mathbf{1}}, x_{0} x_{2} \mathbf{e}^{2}, x_{0} x_{3} \mathbf{e}^{\mathbf{3}}$ behave as vectors under operations while the elements $x_{2} x_{3} \mathbf{e}^{23}, x_{1} x_{3} \mathbf{e}^{31}, x_{1} x_{2} \mathbf{e}^{12}$ behave as angular momentums

\section{Spin states of molecule}

The concept of the nuclear spin symmetry mentioned above has the next detailed content. In case of free molecule there is the rigorous correlated links between the symmetry of rotational wave function and the symmetry of spin function that imposed by the Pauli exclusion principle. The symmetry properties for spin degree of freedom based on an operation of the permutation of spin states of two protons in the spin wave function. The symmetry properties for rotational degree of freedom is a special defined transformation of rotational wave functions that can be presented as the rotation by $\pi$ angle about the symmetry axis of molecule. The paramolecule has the antisymmetric spin function (singlet) and the symmetric rotational wave function. The ortho-molecule vice versa has the symmetric spin functions (triplet) and the antisymmetric rotational wave function. In case of free molecule there are $1_{01}, 1_{10}, 2_{21}, 2_{12}$ ortho-states and $1_{11}, 2_{11}$ para-states.

The transition between the spin states of free molecule and the spin state of free protons occurs due to the dipole-dipole exchange of two protons belonging to given molecule with another third proton that belongs to water molecule also. This molecule denoted as donor was adsorbed on the surface of solid and was strongly held on it, in contrast to the given molecule denoted as acceptor that comes from gas phase. The acceptor molecule is adsorbed on the same surface but for a certain time interval [18]. The dynamical evolution of system composed of three spin moments $s=1 / 2$ leads to the smooth and continuous variation with time the spin system of molecules. We can obtain the spin density matrix of the acceptor or given molecule as function of time if we average the full $8 \times 8$ density matrix over variables of the third proton.

$$
\begin{aligned}
& \rho_{\text {singlet }}= \\
& \frac{1}{4}\left[\begin{array}{cccc}
1-\cos (t)^{2} & 0 & 0 & 0 \\
0 & \cos (t)^{2}+1 & -2 \cos (t)^{2} & 0 \\
0 & -2 \cos (t)^{2} & \cos (t)^{2}+1 & 0 \\
0 & 0 & 0 & 1-\cos (t)^{2}
\end{array}\right] \\
& \rho_{\text {triplet }}= \\
& \frac{1}{12}\left[\begin{array}{cccc}
\cos (t)^{2}+3 & 0 & 0 & 0 \\
0 & -\cos (t)^{2}+3 & 2 \cos (t)^{2} & 0 \\
0 & 2 \cos (t)^{2} & -\cos (t)^{2}+3 & 0 \\
0 & 0 & 0 & \cos (t)^{2}+3
\end{array}\right]
\end{aligned}
$$

where the dimensionless variable $t=\tau \omega$ was provided by the time $\tau$ that molecule spends in adsorption state and the exchange frequency $\omega$ of the third proton with the nearest proton of the acceptor molecule. If $\mathrm{t}=\pi / 2$ then the density matrices $\rho_{\text {singlet }}, \rho_{\text {triplet }}$ receive the diagonal form that is proportional to the unit matrix, corresponding to two fully depolarized spin moments. This is the spin state of latent molecule that is represented by the basis element $\mathbf{e}$ of $\mathcal{G}\left(R^{3,0}\right)$ algebra.

It is known an estimation of the intra-molecular exchange frequency $\nu=2.7 \mathrm{MHz}$ of protons. In the case of intermolecular exchange we have to reduce this value by a factor of 4-5 because the exchange frequency is proportional to $1 / r^{3}$, where $r$ is the distance between protons [18], [17], [20], [19]. The lifetime in adsorption state $\tau$ during which the water molecules will be converted from free into latent and vice versa is about $\sim 1 \mu s$.

The correspondence between the degrees of freedom of water molecule and the elements of $\mathcal{G}\left(R^{3,0}\right)$ algebra is listed in the next table. Energy in $\mathrm{cm}^{-1}$ Rotational Translational Spin

$\begin{array}{cccc}2_{21} & 135.3 & x_{1} x_{2} \mathbf{e}^{\mathbf{1 2}} & x_{0} x_{3} \mathbf{e}^{\mathbf{3}} \\ 2_{11} & 95.2 & x_{3} x_{1} \mathbf{e}^{\mathbf{3 1}} & x_{0} x_{2} \mathbf{e}^{\mathbf{2}} \\ 2_{12} & 79.5 & x_{2} x_{3} \mathbf{e}^{\mathbf{2 3}} & x_{0} x_{1} \mathbf{e}^{\mathbf{1}}\end{array}$

Because the water molecule constitutes the spatial configuration peculiar to the asymmetric rotor having three rotational 
degrees of freedom therefore there are three elements of the reference system with proper coordinates in the second column of table. We can assert the same about the three translational degrees of freedom of molecule having the basis elements with coordinates which are placed in the third column. The spin degree of freedom that is given in the fourth column corresponds to one particle with half-spin in fully depolarized state. It is assumed that we can duplicate the spin state if it needs due to the property of depolarized state. Note, that $\mathcal{G}\left(R^{3,0}\right)$ algebra has 8 basic elements and about the last element $\mathbf{e}^{123}$ we will say below.

\section{Physical properties of latent molecules}

As we have said above, latent molecules have necessarily the reference system or the coordinate system which express the properties and symmetry of the material environment in which molecules are located now or have been immersed previously. The reference system has three planes $\mathrm{e}^{23}, \mathrm{e}^{31}, \mathrm{e}^{12}$ and three vectors $\mathrm{e}^{1}, \mathrm{e}^{2}, \mathrm{e}^{3}$ which constitute the subspaces with dimension of 2 and 1 respectively on the physical 3D-space in accordance with the axioms of $\mathcal{G}\left(R^{3,0}\right)$ algebra. Such subspaces have not any links between themselves [9].

The elements of reference system should be divided into pairs $\left\{\mathbf{e}^{23}, \mathbf{e}^{\mathbf{1}}\right\},\left\{\mathbf{e}^{31}, \mathbf{e}^{\mathbf{2}}\right\},\left\{\mathbf{e}^{\mathbf{1 2}}, \mathbf{e}^{\mathbf{3}}\right\}$ that are composed of the plane and vector which have different indexes. The pairs are separated by the properly defined rotational energy. We denoted pairs by symbols of the rotational level of free molecule $2_{12}, 2_{11}, 2_{21}$ respectively. See the table above. The connections between pairs arise from the condition $x_{0}^{2}+x_{1}^{2}+x_{2}^{2}+x_{3}^{2}=1$ which means that the displacements in all directions are limited.

According to the accepted reference system the latent molecules have to be disposed and move also along the straight lines whose directions are defined by $\mathbf{e}^{\mathbf{1}}, \mathbf{e}^{\mathbf{2}}, \mathbf{e}^{3}$ vectors. The reference system may belong to a given molecule, to a group of molecules or it apply to the whole of gas. The exceptional gas-kinetic properties of latent molecules result in the unusual behavior of the pressure of water vapor if the latent molecules present in gas. The free molecules can transmit the pressure momentum to any of 6 planes that restrict of the vacuum chamber with equal probability. For simplicity we assume that the vacuum chamber has a cubical form and molecules collide with chamber walls only. The latent molecules according to classical rules of motion can transmit the pressure momentum to 2 opposite planes only. This means that the latent molecules can produce the gas pressure three times less than free molecules do. For example, if the water vapor contains half of free and half of latent species then the total pressure has value $2 / 3$ of the pressure in case of free molecules. The temporal variations of the total gas pressure is a good indicator for presence of latent molecules.

The latent molecules have not the radiation transitions between states $2_{12}, 2_{11}, 2_{21}$ for two reasons. According to the accepted model the latent water molecule has not the dipole momentum because the planes $\mathbf{e}^{23}, \mathbf{e}^{31}, \mathbf{e}^{12}$ are associated with the unique geometrical defined plane containing the hydrogen and oxygen atoms. The vectors $\mathbf{e}^{\mathbf{1}}, \mathbf{e}^{2}, \mathbf{e}^{3}$ of the reference system belong to an environment. The given model has not the operator corresponding to an external electrical field. The well-known elements $\mathbf{e}^{01}, \mathbf{e}^{02}, \mathbf{e}^{03}$ of the Clifford algebra [8] corresponding to the components of electrical field belong to another $\mathcal{G}\left(R^{3,1}\right)$ geometric algebra.

The intensity of NMR spectral line of the latent molecules is greater by $1 / 4$ than for free molecules because the protons of latent molecule are considered to be free. The usual or free molecules to be active in NMR spectra have to occupy the triplet spin states that correspond to $3 / 4$ of total number of water molecules. In case of latent molecules all molecules and all protons are active in NMR spectra.

\section{Isomeric non-equilibrium state, experiment}

By now there are a lot of experimental data that are related to the non-equilibrium state of water molecules [21], [22], (this publication is contained also in [24]), [28], [14], [5], [30]. This effect reveals itself via a deviation of the concentration for ortho and para species from the equilibrium value in both directions. Note that the equilibrium state is that when one para-molecule corresponds to three ortho-molecules. This equilibrium concentration can be explained in terms of the nuclear statistical weight due to protons present in the composition of water molecule. In what follows we will denote such state of water molecules as the isomeric non-equilibrium state.

An experimental installation to study the isomeric nonequilibrium of water vapor can be presented in the following way. There is a vacuum chamber with walls that was made from stainless steel, glass or teflon in order to get the relaxation time into isomeric equilibrium state will be sufficiently large. The solid adsorbent in the form of corundum ceramic balls of $2 \mathrm{~cm}$ in diameter are placed into the chamber. The surface of balls was not purified by any technique therefore some atmospheric molecules was adsorbed on surface. Next the chamber is evacuated to the pressure of several $\mathrm{cPa}$ at room temperature and its volume is filled up by water vapor at pressure of 300-500 Pa. There is the measuring system that records the population of rotational levels of free molecules and pressure in chamber as functions of time. We assume that the population of vibrational-rotational states of free molecules are at the equilibrium and the spin isomers of free molecules behave as the different gases due to the strong quantum prohibition for transitions between isomers.

From experiments [22] that was realized on the installation described above one can see that the measured values of population of molecular levels and pressure in chamber become stationary 20-40 minutes later the moment of injection of water vapor into chamber (see Fig. 1). According to the previous interpretation of experiments the pressure decrease is connected with the adsorption on surface of ceramic balls. Now we assume that the pressure decrease give an evidence of the appearance of latent molecules in water vapor.

The interaction of molecules with the surface of adsorbent plays the crucial role in creating latent molecules from free ones and vice versa. Because the energies of rotational levels of free and latent molecules coincide the decisive transformation undergoes the spin system of molecule only according to microscopic mechanism described above.

An overall picture of molecular transformation can be represented by the next way. There are three channels $2_{12}, 2_{11}, 2_{21}$ through which molecules may pass from free (F) state into latent (L) one and vice versa with equal probabilities for FL and LF transitions. If we assume also that the transmission capacity of channels are equal for all channels then the transformation rate of every isomer is proportional to the number of acting channels. The free ortho-molecules have 2 such channels, the para-molecules have 1 channel only.

As we have said above, at the equilibrium three ortho cor- 


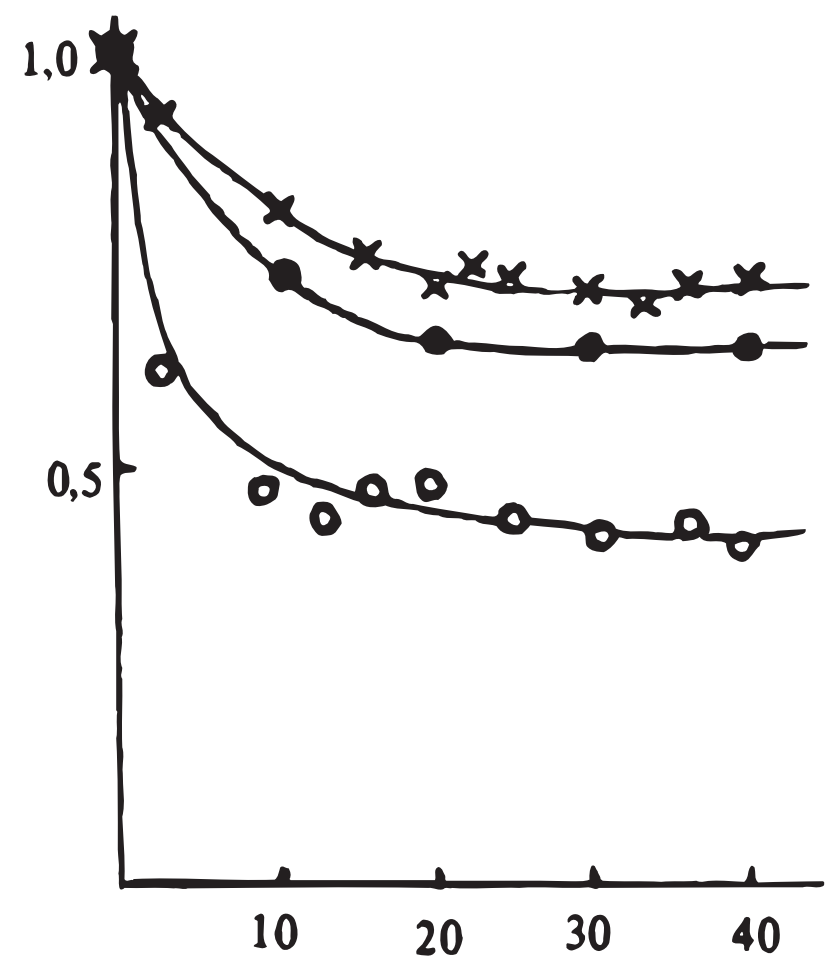

Figure 1. The concentration of ortho-molecules (curve 2), the concentration of para-molecules (curve 1) and the pressure in vacuum chamber (curve 3) as functions of time. All experimental points on Fig. 1 are normalized to the initial value of measured parameters. The time variable is measured in minutes, other parameters are expressed in relative units.

respond to one para species. If we want to keep the othopara ratio equal to 3:1 we have to make available three channels for ortho and one channel for para molecules, but we have just two channels for ortho. This inconsistency between the available channels results in an excess of free orthomolecules in gas. This effect have been observed in all reliable experiments to date.

The process of production of latent molecules can be represented as a gaseous-phase reaction with the solid catalyst and with the reaction rate equal in both directions. The initial substances are the number para-molecule $n_{0}$ and number of ortho-molecules $3 n_{0}$ in volume unit before the reaction starts. It makes sense to introduce the dimensionless variable $x$ to denote the part of para-molecules that is already converted into latent molecules. Then the part of ortho-molecules is $2 x$ because two channels operate. The part of all converted molecules is $3 x$.

The isomeric non-equilibrium state is usually described via the dimensionless variable $\eta$ meaning the ratio of concentrations of ortho to para species

$$
\eta=\frac{3-2 x}{1-x} \quad p / p_{0}=1-\frac{x}{2}
$$

The dynamic equilibrium of interconversion reaction takes place when the concentration of initial reagents is equal to the concentration of reaction products. The concentration of initial reagents is 4 in dimensionless unit therefore its value decreases two times at the dynamic equilibrium. The calculation of the dynamic equilibrium constant $x_{e q}$ using initial reagents or reaction products leads to the coinciding value $x_{e q}=2 / 3$. The pressure of water vapor $p / p_{0}=2 / 3$ in vacuum chamber and the isomeric non-equilibrium ratio $\eta=5$ correspond to this value.
The formula of relative pressure $p / p_{0}$ in vacuum chamber describing the behavior of this parameter as the function of $x$ can be derived by the method that have been used for $\eta$. It should be remembered that the contribution of latent molecules to the overall pressure equals to $1 / 3$ of that value for free molecules. The formula is given in the same row as to $\eta$.

There are three graphs of relative variables as function of time that are typical for two processes that are inherently connected with each other (see Fig. 1). The first process consists in creating of latent molecules and the second one consists in producting of isomeric non-equilibrium state of free molecules. The upper curve (2) is the concentration of free ortho molecules, the next curve (3) is the pressure of water vapor in vacuum chamber, the last curve (1) is the concentration of free para molecules. All variables was normalized to their initial values. To derive the $\eta$ value using these curves the values of upper curve have to be multiplied by 3 . The latent molecules was not fixed independently at that time because we had not any experimental or theoretical ideas about the existence of such objects.

The comparison of experimental data with theoretical predictions shows the quantitative correspondence. The theoretical value of isomeric non-equilibrium $\eta=5$ coincides with the experimental value $5.0 \pm 0.3$ within the experimental error. The experimental value of vapor pressure $0.64 \pm 0.1$ is somewhat lower than theoretical prediction $p / p_{0}=0.67$ due to an uncontrollable adsorption on the surface of vacuum chamber.

\section{Discussion of results}

According to conventional point of view the reason giving rise the effect of isomeric non-equilibrium consists in the spin-selective or isomeric-selective adsorption of water molecules. This means that one of isomers, for example, para isomer, is adsorbed on solid surface with greater probability than ortho isomer. This situation has been realized, for example, in the experiments described above. Such explanation of the effect of non-equilibrium have dominated in our previous publications. Now we refuse from it.

If we observe that free molecules disappear from the rotational or vibrational-rotational spectrum one can assume that vanished molecules was converted in states or phase which can not be registered by given observation method. Before now we considered the unobserved state as adsorbed state only. Now we propose another version of effect of vanishing that is based on the non-ordinary properties of latent molecules. The latent molecules have not rotational quantum transitions on the frequency peculiar to free molecules.

It is known the theoretical interpretation [18] of chromatographic experiments with the aim to achieve the isomeric non-equilibrium state of water molecules in gas [28]. We assumed that the probability of adsorption of two isomers are equal to a first approximation and acting force to produce non-equilibrium is the relatively weak magnetic dipoledipole interaction of protons inside the water molecule. The energy of this interaction is somewhat different for two isomers that influences on the adsorptive lifetime of molecules. However, the chromatographic method shows the effect of non-equilibrium at initial and final moments of process [14] indicating that we deal with another microscopic mechanism. Note that chromatographic separation technique is based on the difference of adsorptive lifetime of molecules which can be separated. 
It is interesting to study the properties of latent molecules based on its electronic structure such as the refractive index for visible light and light scattering by gases. These attributes of latent molecules have not considerable change compared to ones of free molecules. We can perform the demonstration of the reality of existence of latent molecules if we will observe that the corresponding response is constant or nearly constant by appearing isomeric non-equilibrium.

The microscopic conversion mechanism between free and latent molecules remains open from theoretical point of view. The experiments show that not all solid surfaces are capable for generating the isomeric non-equilibrium. This property depends also on the water molecules that strongly hold on surface [23]. The surface interaction changes not only the rotational state of molecule but the spin state also.

The significant problem that is considered in given article are tasks which involve the coordinate system or reference system. Usually the reference system is considered as an auxiliary construction and they are excluded from the final form of theoretical or experimental results. The rotating coordinate system in NMR belongs to this type of objects. If we transfer operators and parameters of the task into such reference system the overall problem becomes more simple because the spin-spin interactions are excluded partially and we can introduce the effective Hamiltonian [33]. In NMR experiments the strong rotating magnetic field makes spectral lines more narrow in condensed material therefore we can not consider the rotating reference system as an abstract object. The general approach to the problem of reference systems is contained in [3].

There is another problem that demands the special study which consists in the comparison of the Lee algebra of Lorentz group with the Lee algebra of geometric algebra $\mathcal{G}\left(R^{3,0}\right)$ that have been derived if we use the commutators or outer product only. Note that these algebras coincide with each other or in other terms are isomorphic. Such study allows to define when the Runge-Lenz vectors have to be considered as relativistic objects with corresponding boost operation or when these vectors are simply elements $\mathbf{e}^{\mathbf{1}}, \mathbf{e}^{\mathbf{2}}, \mathbf{e}^{\mathbf{3}}$ of $\mathcal{G}\left(R^{3,0}\right)$ algebra.

The behavior of latent molecules in condensed phase does not differ essentially from that in gas. The spatial movements of latent molecules are restricted in directions which we can adjust with hydrogen bonds. It is known that the hydrogenbond net may allow the infinitesimal movements without destruction of net [6], [7]. This remark is right if we consider the infinitesimal angle movements in plane.

\section{Conclusion}

We present the most simple model of water molecule with unified low-energy degrees of freedom. This means that the translational, rotational and spin degrees of freedom are unified by one mathematical construction based on geometric algebra $\mathcal{G}\left(R^{3,0}\right)$. We denote a water molecule that is described by given model as latent due to specific physical properties that take place in the rotational and NMR spectra. The translational motion of molecule that is considered classically differs from the usual case also.

The free molecules hold an extensive system of rotational levels that have many "magnetic" substates, if a rotational number are large, corresponding to the projections on the $\mathrm{Oz}$ axis of laboratory coordinate system. On the contrary, the latent molecules have only three rotational levels in accordance to three rotational degrees of freedom of asymmetric rotors and these levels do not degenerate. Note that the degeneracy of rotational energy levels is connected with a symmetry of 3D-space which is described by the $\mathrm{SO}(3)$ group.

The symmetry classification obliged to the spin system of water molecule because there are two fermi-particles with spin $1 / 2$ present in the composition of molecule and the Pauli exclusion principle that demands the antisymmetry for total wave function of quantum system. At the same time it is assumed that spins form an united system that included singlet and triplet states. These states are pure ones but the spin state of latent molecule is the most mixed state corresponding to the non-connected or free spin moments.

The most dramatic events take place with translational degrees of freedom. Each molecule is allowed to move along three directions, more exactly along three straight lines in the 3D-space. The directions of lines may be not orthogonal with each other but their number equals always three that have to agree with the number of translational degrees of freedom of molecule. It is significant that displacements are restricted because the coordinates of movement satisfy the condition $x_{1}^{2}+x_{2}^{2}+x_{3}^{2}=1-x_{0}^{2}$. If coordinates are measured in unit of length then the right-hand of equation has a dimension of square length and it has the sense of physical parameter. Its value can not be defined by mathematical considerations. In dense gas this value defined by the mean free path of molecules, in rarefied gas a reasonable value is about the linear dimension of vacuum chamber, in liquid this value may have the atomic range.

The mathematical structure of $\mathcal{G}\left(R^{3,0}\right)$ algebra allows to predict an existence of quantum state of water molecule that has not an analogy with free molecules. This state corresponds to the basis element $\mathbf{e}^{\mathbf{1 2 3}}$ which is denoted as volume element or as quasiscalar. This basic element arises via the operation of outer multiplication of the plane element with associated vector element. About these structure elements of algebra we have said above.

This new quantum state has a wave function $x_{1} x_{2} x_{3}$ that turn out to be an eigenfunction of the rotational Hamiltonian with the energy $4(\mathrm{c}+\mathrm{b}+\mathrm{a})$ that equals to 206.68 in unit $\mathrm{cm}^{-1}$. This value coincides with the rotational level energy of state $3_{22}$ of free molecule. An unusual property of this state is that we can assign only a single rotational degree of freedom to given state.

It should be instructive to answer the question that has appeared at the beginning of work: how ortho-molecules will be adsorbed? A short answer is next. The free molecule transforms into latent one conserving all degrees of freedom. Being evaporated or desorbed it renews degrees of freedom peculiar to free molecule.

\section{Acknowledgements}

I would like to thank my colleagues S. Borisov and M. Fedorov for many stimulating conversations. 


\section{Appendix A}

An application of the geometric algebra $\mathcal{G}\left(R^{3,0}\right)$ in given study assumes the representation of basis elements by matrices. We choose the most simple case of representation when the Pauli matrices correspond to basis vectors and quaternion matrices correspond to bivectors [1]. All constructions are realized according to [9], [25].

$$
\begin{array}{lcccccccc}
\mathcal{G}\left(R^{0,2}\right) & \mathbf{1} & \multicolumn{1}{c}{\mathbf{i}} & \mathbf{j} & \mathbf{k} \\
\mathcal{G}\left(R^{3,0}\right) & e & e 1 & e 2 & e 3 & e 23 & e 31 & e 12 & e 123 \\
\mathcal{G}^{+}\left(R^{3,1}\right) & e & e 01 & e 02 & e 03 & e 23 & e 31 & e 12 & e 0123 \\
\{e 1, e 2, e 3\} \times e 123 & =\{e 23, e 31, e 12\} \\
\{e 01, e 02, e 03\} \times e 0123 & =\{e 23, e 31, e 12\}
\end{array}
$$

where $\times$ denotes matrices multiplication.

\section{Appendix B}

It is known three 6-fold Lee algebras which are alike on the one hand, but are different in details on the other hand [32], [34], [11]. There are the so(4) algebra of SO(4) group of rotations on the Euclidean 4D-space, the AG algebra which was derived from the geometric algebra $\mathcal{G}\left(R^{3,0}\right)$ if we used commutators of basic elements only and the AL algebra of Lorentz group. We can choose basis of these algebras so that we can divide basis elements of each algebra into sets which consist in two elements. For example, these sets of algebra $\mathcal{G}\left(R^{3,0}\right)$ may be written as

$$
\mathrm{e}^{23}, \mathrm{e}^{1} \quad \mathrm{e}^{31}, \mathrm{e}^{2} \quad \mathrm{e}^{12}, \mathrm{e}^{3}
$$

The second element in each set has only one index instead of two indexes. We can introduce such simplification because the second index of elements mentioned above is always the same. For example, in the case of so(4) algebra we have $a^{14}, a^{24}, a^{34}$.

A general property that makes algebras so(4),AG,AL to be like consists in the fact that the first elements in each set form the Lee subalgebras which are isomorphic to the so(3) algebra of $\mathrm{SO}(3)$ group of rotations on 3D-space. The second elements in each set on the contrary do not form subalgebras. The difference of the so(4) algebra from the AG and AL algebras consists in the sign of commutators of the second elements. The sign of commutators of the second elements coincides with the sign of commutators of the first elements for the so(4) algebra but the AG and AL algebras have the opposite sign as compared to the sign of commutators of the first elements. For example,

$$
\begin{aligned}
& {\left[a^{23}, a^{31}\right]=a^{12} \quad\left[a^{1}, a^{2}\right]=a^{3}} \\
& {\left[\mathbf{e}^{\mathbf{2 3}}, \mathbf{e}^{\mathbf{3 1}}\right]=\mathbf{e}^{\mathbf{1 2}} \quad\left[\mathbf{e}^{\mathbf{1}}, \mathbf{e}^{\mathbf{2}}\right]=-\mathbf{e}^{\mathbf{3}}}
\end{aligned}
$$

If the sign of commutators of the first elements coincides with one of the second elements as for the so(4) algebra we can choose a new basis $a^{23} \pm a^{14}, a^{31} \pm^{24}, a^{12} \pm a^{34}$ in which the so(4) algebra transforms into the direct sum of two subalgebras that are isomorphic to the so(3) algebra. In case of the AG and AL algebras such transformation is impossible. The second elements of the AG and AL algebras correspond with boost transformations in the Lorenz group.

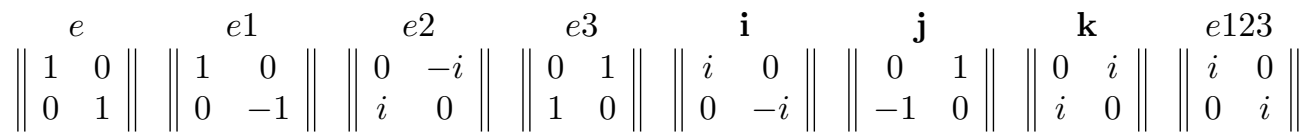

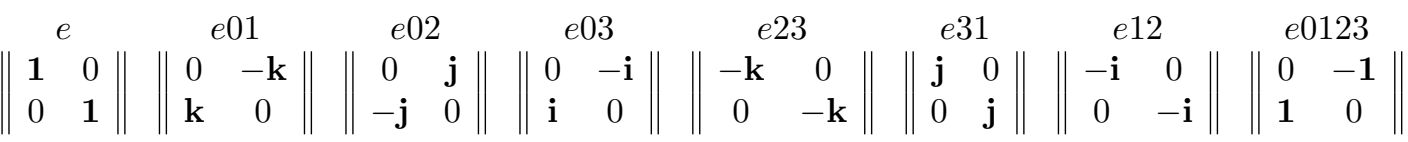

The matrix algebras $\mathcal{G}\left(R^{3,0}\right), \mathcal{G}^{+}\left(R^{3,1}\right)$ are isomorphic because their multiplication tables coincide fully under given correspondence between basis matrices. An application of two isomorphic algebras explains via a fact that the corresponding basis elements have a different physical sense. For example, the elements $e 1, e 2, e 3$ are considered as vectors but the bivectors $e 01, e 02, e 03$ have sense of components of electrical field. 


\section{REFERENCES}

[1] F Berdon Alan. The geometry of discrete groups, 1983.

[2] Ronald Anderson and Girish C Joshi. Quaternions and the heuristic role of mathematical structures in physics. arXiv preprint hep-ph/9208222, 1992.

[3] Stephen D Bartlett, Terry Rudolph, and Robert W Spekkens. Reference frames, superselection rules, and quantum information. Reviews of Modern Physics, 79(2):555, 2007.

[4] Garrett Birkhoff and John Von Neumann. The logic of quantum mechanics. The Annals of Mathematics, 37(4):823-843, 1936.

[5] P Cacciani, J Cosleou, and M Khelkhal. Nuclear spin conversion in h2o. PhysicalReview A, 85(1):012521, 2012.

[6] Martin Chaplin. Water structure and behavior. Avaliable from: www. lsbu. ac.uk/water/hofmeist. html[Links], 2005.

[7] Martin Chaplin. Water structure and science. 1sbu. ac. uk, 2011.

[8] Alexander A Chernitskii. Born-infeld electrodynamics: Clifford number and spinor representations. International Journal of Mathematics and Mathematical Sciences, 31(2):77-84, 2002.

[9] Eric Chisolm. Geometric algebra. arXiv preprint math$\mathrm{ph} / 1205.5935 \mathrm{v} 1,2012$.

[10] Paul C Cross, RM Hainer, and Gilbert W King. The asymmetric rotor ii. Calculation of dipole intensities and line classiffcation. The Journal of Chemical Physics, 12:210, 1944.

[11] IM Gel'fand, RA Minlos, and Z Ya Shapiro. Representations of the rotation group and the Lorentz group. Fizmatgiz, Moscow, page 274, 1958.

[12] Walter Gordy and Robert L Cook. Microwave molecular spectra. John Wiley, 1970.

[13] David Hestenes. Spacetime physics with geometric algebra. American Journal of Physics, 71:691, 2003.

[14] PO Kapralov, VGArtemov,AMMakurenkov, VI Tikhonov, and AA Volkov. Deviations from the normal ortho/para ratio for water (3: 1) in the vapor phase in dynamic sorption. Russian Journal of Physical Chemistry A, 83(4):663-669, 2009.

[15] Gilbert W King, RM Hainer, and Paul C Cross. The asymmetric rotor i. calculation and symmetry classification of energy levels. The Journal of Chemical Physics, 11:27, 1943.

[16] VK Konyukhov. Forward and reverse rotation in the theory of multiatomic molecules. Bulletin of the Lebedev Physics Institute, (5):14-20, 2001.

[17] VK Konyukhov. Mixed state of spin moments of two protons in the water molecule and stability of its spin isomers. Bulletin of the Lebedev Physics Institute, 37(11):359-360, 2010.
[18] VK Konyukhov. Dynamic model of ortho-para conversion of water molecules. Bulletin of the Lebedev Physics Institute, 38(3):76-79, 2011.

[19] VK Konyukhov. Spin states of para-water and ortho-water molecule in gas and liquid phases. Physics and Chemistry of Liquids, 49(3):343-346, 2011.

[20] VK Konyukhov. Proton exchange within water molecule. Bulletin of the LebedevPhysics Institute, 39(8):239-240, 2012.

[21] VK Konyukhov,AM Prokhorov, VI Tikhonov, and VN Faizulaev. Rotationally selective condensation of heavy water in a supersonic carbon dioxide jet. JETP Lett, 43(2):85,1986.

[22] VK Konyukhov, VI Tikhonov, and TL Tikhonova. Spinmodification selective adsorption of water molecules on a surface of corundum ceramic. Kratk. Soobsh. Fiz. FIAN,(9):12-14, 1988.

[23] VK Konyukhov, VI Tikhonov, TL Tikhonova, and VN Faizulaev. Separation of spin-modifications of water and heavy water molecules. PIS'MA ZH. TEKH. FIZ,12(23):1438-1441, 1986.

[24] V.K. Konyukhov (ed.). Physical processes in low-temperature gas-dynamic lasers.1990.

[25] Douglas Lundholm. Geometric algebra and its applications. arXiv preprintmath/0605280, 2006.

[26] Jan A Schouten. Tensor analysis for physicists. DoverPublications. com, 1954.

[27] Malcolm Woodrow Pershing Strandberg. Microwave spectroscopy. Methuen London,1954.

[28] Vladimir I Tikhonov and Alexander A Volkov. Separation of water into its ortho and para isomers. Science, 296(5577):2363-2363, 2002.

[29] Charles Hard Townes. Microwave spectroscopy. DoverPublications. com, 1955

[30] Pierre-Alexandre Turgeon, Patrick Ayotte, Elina Lisitsin, Yossi Meir, Tatyana Kravchuk, and Gil Alexandrowicz. Preparation, isolation, storage, and spectroscopic characterization of water vapor enriched in the ortho-h2o nuclear spin isomer. Physical Review A, 86(6):062710, 2012.

[31] D.A. Varshalovich, A.N. Moskalev, and V.K. Khersonskii. Quantum theory of angular momentum. 1987.

[32] N.Ya. Vilenkin. Special Functions and the Theory of Group Representations..., volume 22. AMS Bookstore, 1968.

[33] JS Waugh. New nmr methods in solid state physics. Cambridge, Massachusetts:Massachusetts Institute of Technology, 1978.

[34] Steven Weinberg and OW Greenberg. The quantum theory of fields, vol. 1: Foundations. Physics Today, 48:78, 1995.

[35] Eugene Wigner. Group theory: and its application to the quantum mechanics of atomic spectra, volume 5. Academic Press, 1959. 